\title{
Primer registro de Branchiomma coheni (Polychaeta: Sabellidae) en las costas del Caribe colombiano
}

\section{First record of Branchiomma coheni (Polychaeta: Sabellidae) along the Colombian Caribbean coast}

\author{
Pedro R. Dueñas-Ramírez ${ }^{1^{*}}$ y Andrea Carolina Dueñas-Lagos ${ }^{2}$
}

\begin{abstract}
RESUMEN
La especie invasora Branchiomma coheni Tovar-Hernández y Knight-Jones, 2006, familia Sabellidae, ha sido recolectada en la Bahía de Cispatá, Morrosquillo y en la Ciénaga Grande de Santa Marta, en las raíces de los mangles y en La Escollera en Gaira sobre recolectores de ostras. Esta especie de la comunidad bentónica cubre las raíces de mangle compartiendo el sustrato con otros invertebrados. Este es el primer registro de B. coheni para el Caribe colombiano.
\end{abstract}

Palabras claves: Sabélidos, poliquetos, invasor, Caribe, Colombia.

\begin{abstract}
The non-indigenous sabellid polychaete Branchiomma coheni Tovar-Hernández and Knight-Jones, 2006, has been collected in the estuarine waters of Morrosquillo at Bahía Cispatá, in Ciénaga Grande de Santa Marta on mangrove roots, and in La Escollera, Gaira, in oyster spat collectors. This species is representative of a fouling community in mangrove roots and lives together with other invertebrates. This is the first record of B. coheni from the Caribbean coast of Colombia.
\end{abstract}

Keywords: Sabellid, polychaete, invader, Caribbean, Colombia.

\section{INTRODUCCIÓN}

Entre los poliquetos que hacen parte de la biocenosis del bentos de aguas estuarinas tropicales, se encuentran los miembros de la familia Sabellidae, los cuales logran fijarse y construir tubos blandos, con mucus y limo, sobre boyas, pilotes de muelles, rocas cubiertas de esponjas y/o ascidias y raíces de mangle. Seis especies del género Branchiomma han sido registradas para las costas del Caribe y Pacífico de Panamá (Tovar-Hernández \& Knight-Jones, 2006). Para el Caribe colombiano en los manglares de Cartagena, Cispatá, Ciénaga Grande de Santa Marta (CGSM) y Santa Marta se han reportado los sabélidos Sabellastarte magnifica, Bispira brunnea, Parasabella microphtalmus y Branchiomma nigromaculatum (Dueñas, 1980) como parte del bentos litoral, siendo esta última especie la que mejor representa a la familia debido a su abundancia en las raíces de mangle (Dueñas, 2005). Sin embargo, un nuevo representante del género

\footnotetext{
Universidad de Bogotá, Jorge Tadeo Lozano, sede Santa Marta, pedror.duenasr@utadeo.edu.co*

Instituto de Investigaciones Marinas y Costeras, José Benito Vives, INVEMAR, Salguero, Santa Marta, ancadula@gmail.com
}

Recibido: 4 de julio de 2016

Corregido: 12 de setiembre de 2016

Aceptado: 13 de setiembre de 2016

DOI: http://dx.doi.org/10.15359/revmar.8-2.7 
Branchiomma ha sido recolectado recientemente en el 2015, en material procedente del antiguo estuario del Río Sinú, en Cispatá, Córdoba. Al final de ese año se recolectaron varios ejemplares en la pequeña laguna costera de La Escollera, zona urbana de El Rodadero, en Santa Marta, Magdalena. Posteriormente, en el 2016 se recolectó la especie en el manglar del sector Tasajera en la Ciénaga Grande de Santa Marta. En este estudio, esa especie de sabélido se identificó como Branchiomma coheni Tovar-Hernández \& Knight-Jones y constituye el primer registro de la especie para el Caribe.

\section{MATERIALES Y MÉTODOS}

En el sector sur del Caribe colombiano se encuentra la zona estuarina de Cispatá, con una superficie aproximada de $130 \mathrm{~km}^{2}$ localizada en los municipios de San Antero, San Bernardo del Viento y Lorica, departamento de Córdoba; comprende el área correspondiente al antiguo delta del Río Sinú. Varias islas del sector de Soledad, Caño Mocho, el Garzal, Navío y Nisperal están cubiertas de mangle (Olaya et al. 1987) (Fig. 1 A). Más al norte y al oriente del Río Magdalena el manglar cubre el Vía-Parque Nacional Natural Isla de Salamanca y un área mayor sobre la ribera occidental de la Ciénaga Grande de Santa Marta (CGSM). Toda el área, entre manglar, cuerpo de agua y caños, tiene 85.000 ha. Algunas islas dentro de la CGSM, en el complejo de Pajaral, en el Santuario de Flora y Fauna de la CGSM y en la zona Caño Clarín-Mendegua, también se ha- llan cubiertas por mangle (HernándezCamacho et al. 1980) (Fig. 1 B). Más hacia el norte en la zona urbana del balneario El Rodadero, en Santa Marta, se encuentra una marina con acceso a la Ensenada de Gaira a través de un canal, que convierte a este cuerpo de agua en una pequeña laguna costera (Fig. 1 C) rodeada de manglar (Rhizophora mangle), con una profundidad promedio de $2 \mathrm{~m}$ (Franco, 2005), donde el recambio de agua se logra gracias a la acción de la marea. En los lugares de recolección los organismos se encontraron entre los manglares cuyas raíces sumergidas tienen una variada comunidad de macroalgas e invertebrados, tales como ostras, esponjas, ascidias, cirripedios y anémonas, entre otros invertebrados que conforman conglomerados (fouling). En el caso de los individuos recolectados en

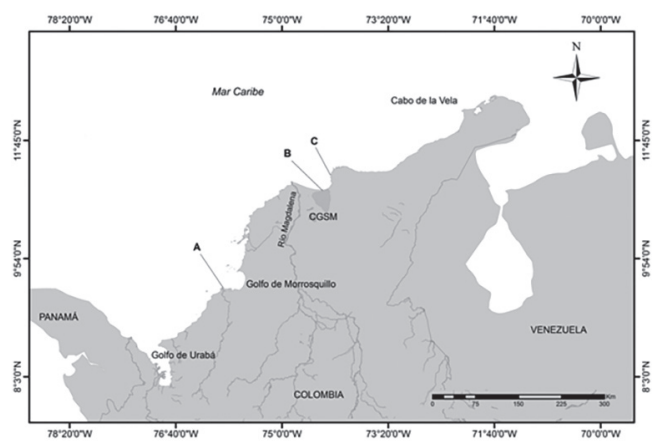

Fig. 1. Ubicación de los tres sitios donde se ha encontrado Branchiomma coheni. A) Bahía de Cispatá, B) Ciénaga Grande de Santa Marta - Tasajera, C) Ensenada de Gaira - La Escollera. Elaborado con ArcGIS 10.1

Fig. 1. Location of the three collection sites of Branchiomma coheni. A) Cispatá Bay, B) Ciénaga Grande de Santa Marta - Tasajera, C) Ensenada de Gaira - La Escollera. Prepared with ArcGIS 10.1 
Santa Marta, se separaron de recolectores de ostras. La toma de muestras se hizo de forma manual, retirando masas de conglomerados de invertebrados sobre las raíces. Al separar en detalle sus componentes se hicieron evidentes los tubos de sabélidos que fueron fijados con formalina al $4 \%$ y llevados al laboratorio para su análisis. Las fotografías se lograron con equipo de microfotografía Nikon. Los seis individuos recolectados se depositaron en la colección de invertebrados de la Universidad de Bogotá, Jorge Tadeo Lozano, sede Santa Marta, con la catalogación UBIAn 024, UBIAn 025 y UBIAn 026.

\section{RESULTADOS}

\section{Sistemática:}

Familia Sabellidae Latreille, 1825

Género Branchiomma Kölliker, 1858

Especie Branchiomma coheni Tovar-Hernández and KnightJones, 2006,

Tovar-Hernández (2009). 507-508., Keppel et al. (2015), Fig. 3 a - d. 499-518

Material examinado: UBIAn 024 (2), 19 Jun/15, Bahía de Cispatá (Córdoba), 9० 23' 3.38' N - 75 47' 30" O; UBIAn 025 (2), 14 Nov/15, La Escollera (Magdalena), $10^{\circ} 58^{\prime} 35^{\prime \prime} \mathrm{N}$ - 74 20' 07" O; UBIAn 026 (2), 10 Abr/16, Tasajera (Magdalena), $11^{\circ} 12^{\prime}$ $33^{\prime \prime} \mathrm{N}-74^{\circ} 13^{\prime} 30^{\prime \prime} \mathrm{O}$.

Diagnosis: Branchiomma coheni en principio no tiene un tubo que la diferencie fácilmente de otros sabélidos.
Todo su cuerpo de color café está salpicado con pequeñas manchas negras. En los individuos recolectados en la Bahía de Cispatá la longitud del cuerpo es 1.8 y $1.9 \mathrm{~cm}$ y de la corona 0.7 y $1.0 \mathrm{~cm}$, respectivamente, mientras que en los recolectados en La Escollera la longitud corporal es de 1.6 y $2.0 \mathrm{~cm}$ y sus coronas con 0.8 y $1.2 \mathrm{~cm}$, finalmente, los ejemplares de CGSM-Tasajera presentan cuerpos de 2.0 y $2.3 \mathrm{~cm}$ y coronas de 1.0 y $1.2 \mathrm{~cm}$. En la corona tiene manchas de color naranja, asociadas a los pares de manchas oculares y los pares de estilodos dispersos a lo largo de todos los radiolos (Fig. 2a). Los pares de estilodos no son de igual longitud, uno es más corto que el otro (Fig. 2c) y en la mitad de cada radiolo, presentan de tres a cuatro pares de los estilodos ensanchados (Fig. 2b), con aspecto foliar y hacia el extremo anterior muestran un borde aserrado. Los lóbulos ventrales del collar son triangulares. El collar con lóbulos ventrales triangulares tiene una mancha naranja en su parte interna. Presenta ocho segmentos torácicos, sobre los cuales llevan filas de uncinos de forma avicular con dos filas de dientes pequeños sobre el gancho principal (Fig. 2d). En cada segmento hay ocelos interramales entre el notopodio y neuropodio, a cada lado del cuerpo.

\section{DISCUSIÓN}

Branchiomma coheni fue descrita para el Pacífico de Panamá. Posteriormente fue reportada para la costa de Baja California, en el Pacífico de México y Keppel et al. (2015) informaron su presencia en el Atlántico occidental, concretamente en la Bahía 

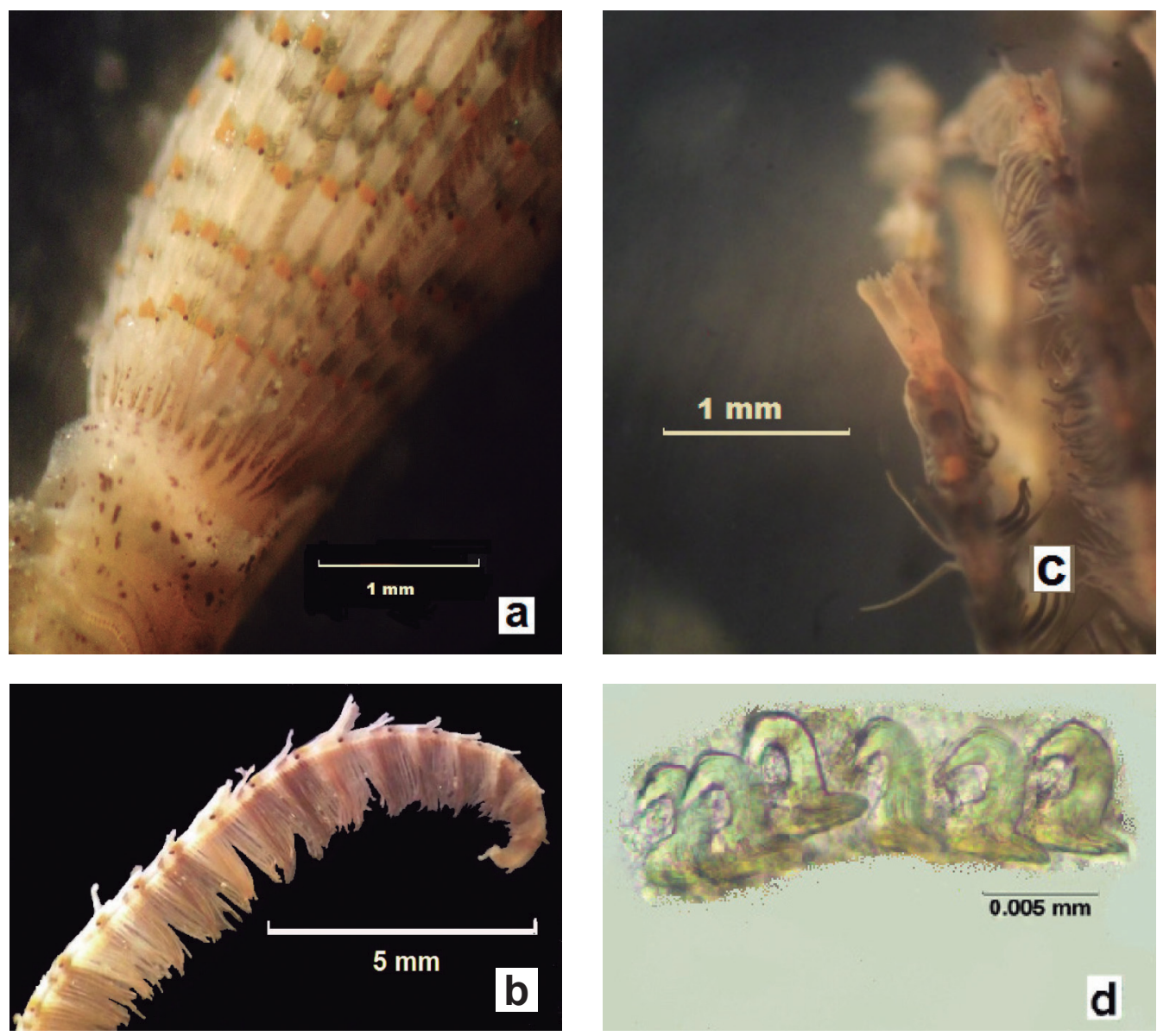

Fig. 2. Caracteres diagnósticos de B. coheni. a) Detalle del collar y corona branquiotentacular. b) Lóbulos ventrales triangulares. c) Estilodos foliares. d) Uncinos aviculares torácicos

Fig. 2. Morphological features of B. coheni. a) Detail of collar and branchial filaments, b) Triangular ventral lobes, c) Foliose stylodes, d) Thoracic avicular uncini

de Tampa, en Florida. Este es el primer hallazgo para el Caribe en Colombia dentro de la Bahía de Cispatá, antiguo delta del Río Sinú en Córdoba, en la Ciénaga Grande de Santa Marta y en La Escollera, área urbana de El Rodadero en Santa Marta. No existen registros de la especie en localidades entre Panamá y Florida, pero es muy probable que la especie haya logrado su establecimiento en otras localidades caribeñas. Capa et al. (2013) realizaron un estudio molecular de Branchiomma para Australia basados en dos especies confirmadas, pero sorpresivamente encontraron ocho que se habían registrado como definidas para otras regiones y países. Entre ellas, B. bairdi considerada originaria del Atlántico y presente además en Hawái. Este 
género ha sido reportado en diferentes partes del mundo con un número considerable de especies invasoras. A partir de su aparición como especie introducida en Florida, ahora son necesarios estudios sobre su ecología y monitoreo en Colombia.

\section{AGRADECIMIENTOS}

Los autores agradecen a Paulo Tigreros por la revisión y comentarios al manuscrito. A la Universidad de Bogotá, Jorge Tadeo Lozano, sede Santa Marta, por el apoyo con el equipo de microfotografía. A Roberto Lemaitre por su colaboración para la recolecta de muestras y a los evaluadores de la revista.

\section{BIBLIOGRAFÍA}

ArcGIS 10.1 (2011). ArcGIS [software GIS]. Redlands, CA: Environmental Systems Research Institute, Inc.

Capa, M., Pons, J. \& Hutchings, P. (2013). Cryptic diversity, intraspecific phenetic plasticity and recent geographical translocations in Branchiomma (Sabellidae, Annelida). Zool. Scripta, 42(6), 637-655.

Dueñas, P. R. (1980). Inventario preliminar de los poliquetos (Annelida) de aguas someras de la Bahía de Cartagena y áreas adyacentes. B. Museo del Mar, 10, 82-138.

Dueñas, P. R. (2005, octubre). Poliquetos (Annelida) del litoral Caribe, noreste del Departamento de Córdoba - Colombia. Ponencia presentada en el XL Congreso Nacional de Ciencias Biológicas, Cali, Colombia.

Franco, A. (2005). Oceanografia de la Ensenada de Gaira: El Rodadero más que un centro turístico en el Caribe colombiano. Bogotá D.C., Colombia: Fundación
Universidad de Bogotá, Jorge Tadeo Lozano.

Hernández-Camacho, J. I., von Hildebrand, P. \& Álvarez-León, R. (1980). Problemática del manejo de manglares con especial referencia al sector occidental de la Ciénaga Grande de Santa Marta. Magdalena. Colombia. En H. Sánchez-Páez \& R. Álvarez-León (Eds.), Diagnóstico $y$ zonificación preliminar de los manglares del Caribe colombiano (pp. 365386). Bogotá D.C., Colombia: Ministerio de Medio Ambiente y Organización Internacional de Maderas Tropicales.

Keppel, E., Tovar-Hernández, M.A. \& Ruiz, G. (2015). First record and establishment of Branchiomma coheni (Polychaeta: Sabellidae) in the Atlantic Ocean and review of non-indigenous species of the genus. Zootaxa, 4058(8), 499-518. http:// dx.doi.org/10.11646/zootaxa.4058.4.3

Olaya, H., Centenaro, D., Leguízamo, I. \& Pineda, F. (1987). Los bosques de mangle del antiguo delta del Río Sinú (Córdoba-Colombia). Estudio de la vegetación y aprovechamiento. Montería, Colombia: Corporación Autónoma de los Valles del Sinú y San Jorge, CVS.

Tovar-Hernández, M. A. \& Knight-Jones, P. (2006). Species of Branchiomma (Polychaeta: Sabellidae) from the Caribbean Sea and Pacific coast of Panama. Zootaxa, 1189, 1- 37.

Tovar-Hernández, M. A. (2009). Sabellidae Latreille, 1825. En J. A. de LeónGonzález, J. R. Bastida-Zabala, L. F. Carrera-Parra, M. E. García-Garza, A. Peña-Rivera, S. J. Salazar-Vallejo \& V. Solís-Weiss (Eds.), Poliquetos (Annelida: Polychaeta) de México y América Tropical (pp. 489-520). Nuevo León, Monterrey, México: Editorial Universidad Autónoma de Nuevo León. 